\title{
Scanning Ion Conductance Microscopy for Single Cell Analysis
}

Petr Gorelkin ${ }^{1}$, Alexander Erofeev ${ }^{2}$, Andrew Shevchuk ${ }^{3}$, Pavel Novak ${ }^{2}$, Christopher Edwards ${ }^{4}$ and Yuri Korchev $^{3}$

${ }^{1}$ Medical Nanotechnology; National University of Science and Technology MISiS, Moscow, Moskva, Russia, ${ }^{2}$ National University of Science and Technology "MISIS"; Lomonosov Moscow State University, Moscow, Moskva, Russia, ${ }^{3}$ Imperial College London, London, England, United Kingdom, ${ }^{4}$ ICAPPIC Ltd, London, England, United Kingdom

In the last few decades a micropipette has become an irreplaceable tool in cell physiology. It has been widely used for local application of chemicals, rapid perfusion application, intra- and extracellular perfusions and voltage measurements, whole cell and single channel current recording, etc. Many breakthrough discoveries leading to a better understanding of cell physiology and cell functions have been made using this tool. The functionality of this rather simple tool has been dramatically expanded with miniaturisation of the pipette tip down to nanometre scale, and invention of scanning ion conductance microscopy (SICM). SICM uses ion current flowing out of the tip of a glass nanopipette (pipette with tip diameter $<100 \mathrm{~nm}$ ) filled with electrolyte to detect presence of a surface in a noncontact fashion. By scanning the sample in $\mathrm{x}-\mathrm{y}$ plane and moving the nanopipette up and down on the $\mathrm{z}$-axis the SICM recreates $3 \mathrm{D}$ topography image of the sample surface. We have shown that scanning nanopipettes are capable of reproducing 3D topography of complex, soft, live biological samples such as neuronal networks or inner ear hair cells (Novak et al., 2009) at resolution better than atomic force microscopy.

The SICM probe a mico- or nanopipette is ideally suited for performing nanoscale assays on the cell surface. These include patch-clamp recording from individual surface structures, iontophoretic delivery of reagents, or pressure micro-application via the pipette to probe mechanical properties of the cell or to deliver reagents. Until now, these advantages of SICM have never been fully explored in such important preparations as brain slices or cell cultures with a complex surface topology because the requirement for a relatively flat specimen surface has been intrinsic to standard scanning probe techniques.

To circumvent these difficulties, we have developed "hopping-mode" ion conductance microscopy. This methodology allows us to image uneven and convoluted cell surfaces at high resolution by ensuring that the pipette always approaches the cell from above rather than 'dragging' along its surface (Novak et al., 2009). Figure 1 shows an image of a dissociated hippocampal culture rendered using this new method, with a pipette of inner radius of $50 \mathrm{~nm}$. We can clearly resolve fine dendritic segments, even those "suspended" in space. In order to unambiguously identify synaptic connections, we combined this new imaging mode with fluorescence imaging.

We have successfully combined scanning nanopipettes with principles of electrophysiology, fluorescence microscopy, electrochemistry (Erofeev et al., 2018), electrophoresis (Zhang et al., 2019), and electroosmosis to investigate single channels and receptors in cellular membranes, to deliver biomolecules to precisely defined locations in cell cultures, and to perform single cell analysis (Akasov et al., 2019). With recent progress in nanopore-based biosensing and sequencing, and also nanopipette-based 3D printing we are only just beginning to open a new window into the life at nanoscale and realising new possibilities for engineering of interfaces between single cells and man-made electronics for therapeutic and diagnostic purposes or synthesis of entirely novel bioelectrical circuits. 
a)

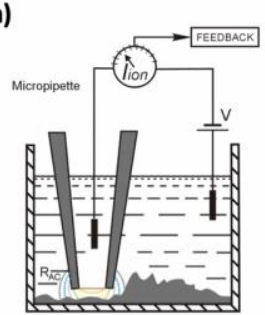

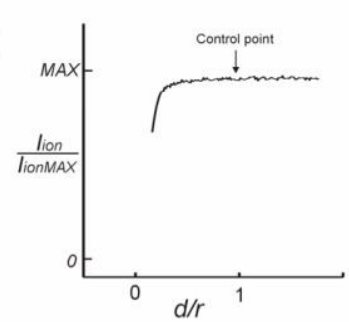

c)

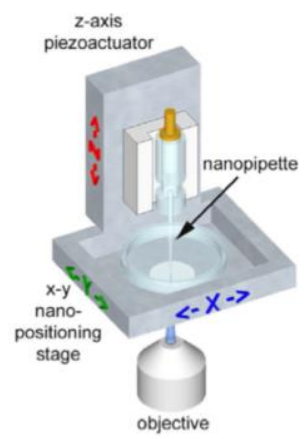

b)

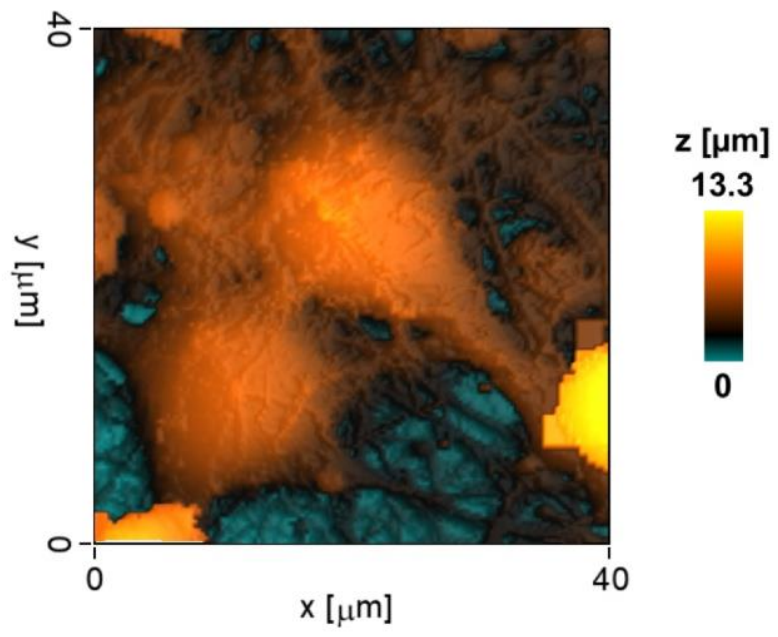

Figure 1. a) Scheme of feedback control of SICM, b) Topography image SICM image of live neuron from mouse hippocamp, c) Scheme of SICM scanning system.

\section{References}

Akasov, R. A., Sholina, N. V., Khochenkov, D. A., Alova, A. V., Gorelkin, P. V., Erofeev, A. S., Generalova, A. N. \& Khaydukov, E. V. (2019). Photodynamic therapy of melanoma by blue-light photoactivation of flavin mononucleotide. Scientific Reports 9, 9679. http://www.nature.com/articles/s41598-019-46115-w.

Erofeev, A., Gorelkin, P., Garanina, A., Alova, A., Efremova, M., Vorobyeva, N., Edwards, C., Korchev, Y. \& Majouga, A. (2018). Novel method for rapid toxicity screening of magnetic nanoparticles. Scientific Reports 8, 7462. http://www.nature.com/articles/s41598-018-25852-4.

Novak, P., Li, C., Shevchuk, A. I., Stepanyan, R., Caldwell, M., Hughes, S., Smart, T. G., Gorelik, J., Ostanin, V. P., Lab, M. J., Moss, G. W. J., Frolenkov, G. I., Klenerman, D. \& Korchev, Y. E. (2009). Nanoscale live-cell imaging using hopping probe ion conductance microscopy. Nature Methods 6, 279281. http://www.nature.com/articles/nmeth.1306.

Zhang, Y., Takahashi, Y., Hong, S. P., Liu, F., Bednarska, J., Goff, P. S., Novak, P., Shevchuk, A., Gopal, S., Barozzi, I., Magnani, L., Sakai, H., Suguru, Y., Fujii, T., Erofeev, A., Gorelkin, P., Majouga, A., Weiss, D. J., Edwards, C., Ivanov, A. P., Klenerman, D., Sviderskaya, E. V., Edel, J. B. \& Korchev, Y. (2019). High-resolution label-free 3D mapping of extracellular $\mathrm{pH}$ of single living cells. Nature Communications 10, 5610. http://www.nature.com/articles/s41467-019-13535-1.

Acknowledgements.

Electrochemical measurements of ROS level were carried out with financial support from the Ministry of Education and Science of the Russian Federation in the framework of increase Competitiveness Program of NUST "MISIS", implemented by a governmental decree dated 16th of March 2013, No 211. SICM experiments were supported by the Russian Science Foundation 19-79-30062. 